\title{
DESENVOLVIMENTO DE ESPÉCIES ARBÓREAS NATIVAS EM UMA ÁREA REFLORESTADA DO CORREDOR DE BIODIVERSIDADE SANTA MARIA-PR ${ }^{1}$
}

\author{
Rosimeri de Oliveira Fragoso², Lívia Godinho Temponi ${ }^{3}$, Ana Tereza Bittencourt Guimarães ${ }^{4}$ e Andreia \\ Kuusmota Bonini ${ }^{5}$
}

\begin{abstract}
RESUMO - Objetivou-se avaliar um plantio de enriquecimento com 12 espécies arbóreas nativas da região, em uma área reflorestada do Corredor de Biodiversidade Santa Maria, localizado entre os Municípios de Santa Terezinha de Itaipu e São Miguel do Iguaçu, no Paraná. Objetivou-se, também, avaliar a persistência da espécie invasora Megathyrsus maximus (Jacq.) B. K. Simon \& S. W. L. Jacobs, conhecido como capim-colonião, e alterações nas características físicas e químicas do solo. As avaliações consistiram no acompanhamento mensal da mortalidade e desenvolvimento das mudas e da massa de matéria seca obtida no manejo do capim-colonião. As alterações promovidas no solo foram verificadas por meio de três coletas de solo ao longo do experimento. A mortalidade alcançou $24,38 \%$, sendo mais acentuada nos dois primeiros meses, janeiro $(11,88 \%)$ e fevereiro (5,63\%). Em relação ao desenvolvimento das mudas, destacaram-se as espécies Aloysia virgata (Ruiz \& Pav.) Juss. (lixeira) e Ceiba speciosa (A. St.-Hil.) Ravenna (paineira), com acréscimos mensais em diâmetro de 0,80 mm e $0,60 \mathrm{~mm}$ e em altura de 5,80 cm e $1,74 \mathrm{~cm}$, respectivamente. O controle do capim-colonião pela metodologia aplicada foi eficiente, não havendo reincidência considerável ao longo dos meses. Por meio das análises de solo, foi possível observar que a recuperação da área, mesmo que por período curto, mostrou-se eficiente na alteração das características físicas e químicas do solo, podendo ainda levar a novas modificações em decorrência do desenvolvimento das mudas.
\end{abstract}

Palavras-chave: Corredor ecológico; Restauração ambiental; Avaliação de espécies.

\section{DEVELOPMENT OF TREE NATIVE SPECIES IN A REFORESTED AREA AT THE BIODIVERSITY CORRIDOR SANTA MARIA-PR}

\begin{abstract}
This study aimed to evaluate an enrichment planting of 12 tree species native to the region, in a reforested area of the Biodiversity Corridor Santa Maria, located between the cities of Santa Terezinha de Itaipu and São Miguel do Iguaçu-PR. This study also aimed to evaluate the persistence of the invasive species Megathyrsus maximus (Jacq.) B. K. Simon \& S. W. L. Jacobs, known as green panic grass, and it also monitored changes in physical and chemical characteristics of the soil. The evaluations consisted of monthly monitoring of the mortality rate and seedling growth and dry mass obtained from the management of green panic grass. The changes introduced in the soil were checked using three soil samples throughout the experiment. Mortality reached a percentage of $24.38 \%$, which was more intense in the first two months: January (11.88\%) and February (5.63\%). Regarding the development of seedlings, the species that stood out were Aloysia virgata (Ruiz \& Pav.) Juss. (sweet almond verbena), and Ceiba speciosa (A.St.-Hil.) Ravenna (silk floss tree), with monthly increases in diameter of $0.80 \mathrm{~mm}$ and $0.60 \mathrm{~mm}$, and in height of $5.80 \mathrm{~cm}$ and $1.74 \mathrm{~cm}$, respectively. The control of green panic grass by the applied methodology was efficient, with
\end{abstract}

\footnotetext{
${ }^{1}$ Recebido em 15.05.2013 aceito para publicação em 17.09.2014.

${ }^{2}$ Programa de Pós-Graduação em Conservação e Manejo de Recursos Naturais, Universidade Estadual do Oeste do Paraná, UNIOESTE, Brasil. E-mail: <meri_ol@yahoo.com.br>.

${ }^{3}$ Centro de Ciências Biológicas e da Saúde, Universidade Estadual do Oeste do Paraná, UNIOESTE, Brasil. E-mail: <liviatemponi@yahoo.com.br>.

${ }^{4}$ Centro de Ciências Biológicas e da Saúde, Universidade Estadual do Oeste do Paraná, UNIOESTE, Brasil. E-mail: <anat@brturbo.com.br> .

${ }^{5}$ Centro de Ciências Biológicas e da Saúde, Universidade Estadual do Oeste do Paraná, UNIOESTE, Brasil. E-mail:

<akbonini@yahoo.com.br>.
} 
no significant recurrence over the months. Through soil analysis, it was possible to observe that the recovery of the area, even for a short period, proved to be effective in altering the physical and chemical characteristics of the soil, and it may also lead to new modifications due development of seedlings.

Keywords: Ecological corridor; Environmental restoration; Species selection.

\section{INTRODUÇÃO}

O Bioma Mata Atlântica é composto por um complexo de diferentes formações florestais caracterizadas por áreas de alta biodiversidade e endemismo, o que confere a esse domínio identidade biogeográfica particular (GALINDO-LEAL; CÂMARA, 2003). Fazem parte da composição original desse bioma vegetações definidas como florestas ombrófilas densas, abertas e mistas, florestas estacionais decidual e semidecidual, campos de altitude, mangues e restingas (SILVA; CASTELETI, 2003).

Além da complexidade de paisagens, uma multiplicidade de fatores socioeconômicos contribui para um mosaico de situações biológicas e sociais, estando a Mata Atlântica como um dos biomas mais ameaçados do mundo (RIBEIRO et al., 2009).

Tal pressão exercida sobre as Floretas Tropicais, devido à fragmentação de paisagens e inserção destas em matrizes não florestais, é um dos grandes problemas da conservação da biodiversidade. O isolamento tende a dificultar o fluxo gênico entre populações de espécies vegetais e animais, podendo comprometer a conservação desses locais em longo prazo (GROENEVELDA et al., 2009; PÜTZ et al., 2011).

Com isso, práticas que visam reconectar fragmentos de remanescentes florestais têm sido realizadas, e estratégias abrangendo a proteção do entorno dessas áreas têm sido formuladas, visando permitir a gestão de cenários inteiros (GANEM, 2011).

Essas áreas de conexão são chamadas de corredores ecológicos, que, por sua vez, funcionam como faixas de cobertura vegetal que permitem o fluxo de espécies de uma área para outra, podendo criar fontes de regeneração natural que contribuirão para a recuperação mais rápida do ambiente (VOS et al., 2002). A regeneração natural, quando possível, possibilita a entrada no ecossistema de espécies já bem adaptadas à dinâmica sucessional, com maiores taxas de sucesso em relação ao seu estabelecimento (CABIN et al., 2002). Contudo, muitas dessas áreas passaram por processos de degradação extensivos e estão situadas longe de matrizes florestais que poderiam atuar como fonte de propágulos, limitando sua dispersão e colonização. Nesses casos, outras medidas são necessárias, e a entrada de espécies no ambiente fica dependente de intervenções antrópicas como plantio de mudas (MARTINS, 2007). A recomposição florestal por meio do plantio requer o emprego de técnicas adequadas, definidas de acordo com avaliações detalhadas do ambiente e tipo de perturbação.

De maneira geral, espécies de ocorrência natural em determinado local podem ter mais sucesso devido à presença na área de seus polinizadores e dispersores naturais, garantindo, assim, que elas tenham sua reprodução e regeneração facilitadas (DAEHLER, 2003). Além disso, o manejo durante e após o plantio contribui para que os esforços empregados na restauração de uma área atinjam seus objetivos. O controle de espécies competidoras de gramíneas, por exemplo, é com frequência um trabalho que exige acompanhamento prolongado (HOLL, 2002; MONACO; SHELEY, 2012).

O adensamento da área com espécies arbóreas, visando promover maior sombreamento, pode auxiliar no controle de gramíneas e, assim, no estabelecimento de espécies nativas. Da mesma forma, o enriquecimento ajuda a restaurar a diversidade e acelerar a sucessão da floresta (RODRIGUES et al., 2009).

Análises do solo são importantes em projetos de reflorestamento, pois fornecem informações sobre as condições estruturais e nutricionais da área que servirão de base para o crescimento das plantas (NEGREIROS et al., 2011). Análises periódicas, por sua vez, ajudam a compreender as transformações ocorridas ao longo do tempo.

Dessa forma, objetivou-se avaliar o desempenho de 12 espécies arbóreas utilizadas para o enriquecimento da área e a persistência da espécie invasora Megathyrsus maximus sob o manejo mensal no sub-bosque de uma área reflorestada de corredor ecológico. Além disso, objetivou-se verificar possíveis alterações do solo,

Revista Árvore, Viçosa-MG, v.38, n.6, p.1003-1013, 2014 
quanto às suas características físicas e químicas, em relação ao plantio das mudas e manejo da espécie Megathyrsus maximus, visando subsidiar projetos de restauração em condições ambientais semelhantes.

\section{MATERIAL E MÉTODOS}

\section{1. Área de Estudo}

A área de estudo está situada no Corredor de Biodiversidade Santa Maria, localizado entre os Municípios paranaenses de Santa Terezinha de Itaipu e São Miguel do Iguaçu, o qual é responsável pela junção entre o Parque Nacional do Iguaçu e a Faixa de Proteção do Reservatório de Itaipu.

O corredor abrange as Bacias Hidrográficas dos Rios Iguaçu e Paraná III (TOSSULINO et al., 2007), localizado no terceiro planalto paranaense, com formação dos solos principalmente Latossolo Vermelho e Nitossolo (MINEROPAR, 2013). A classe textural do solo foi determinada como Franco Siltosa, com cerca de 20\% de argila, $22 \%$ de areia e $58 \%$ de silte, baseando-se no diagrama textural proposto pelo USDA (FREIRE, 2006). A Região Fitoecológica é Floresta Estacional Semidecidual (IBGE, 2012) e o clima, Subtropical Úmido Mesotérmico, classificado por Köppen como Cfa, com verões atingindo temperatura média superior a $22^{\circ} \mathrm{C}$ e invernos com média inferior a $18^{\circ} \mathrm{C}$, numa temperatura média anual de $21^{\circ} \mathrm{C}$. A precipitação varia em torno de $1.800 \mathrm{~mm}$, com chuvas distribuídas durante o ano, mas com pequena redução no inverno (IAPAR, 2011).

O ponto de implantação do experimento está situado nas coordenadas 25²9’02.62' Sul e 54²1'17.10'” Oeste, em uma faixa que foi reflorestada em 2003 predominantemente com espécies arbóreas pioneiras nativas da região (Figura 1). Contudo, por ser uma faixa estreita, está sujeita a efeitos de borda, como a presença da gramínea exótica Megathyrsus maximus (Jacq.) B. K. Simon \& S. W. L. Jacobs, conhecida popularmente como capim-colonião.

\subsection{Implantação do Projeto}

Foram utilizadas oito parcelas de 10 x $10 \mathrm{~m}\left(100 \mathrm{~m}^{2}\right)$, dispostas em duas linhas paralelas, nas quais se realizou o corte do capim-colonião mensalmente, deixando-o sobre o solo como cobertura morta.

As mudas foram plantadas de acordo com o modelo de repovoamento florestal em linhas, com 20 mudas

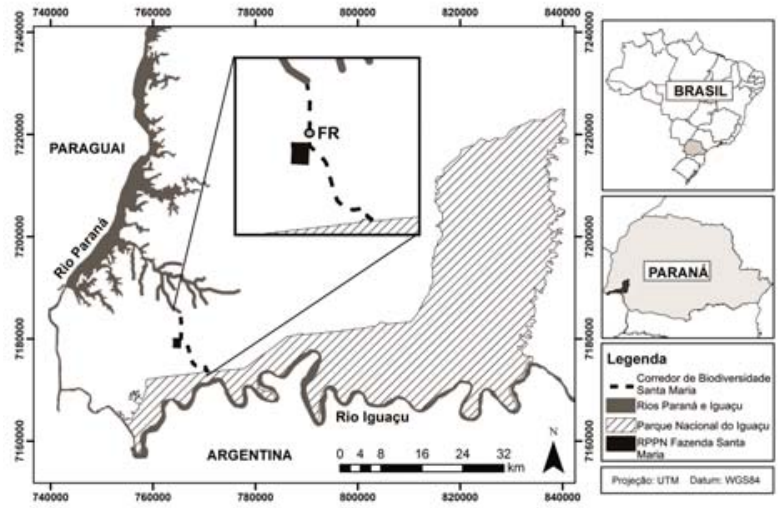

Figura 1- Localização da área de estudo no Corredor de Biodiversidade Santa Maria, PR. FR: faixa reflorestada em 2003.

Figure 1-Location of the study area at the Biodiversity Corridor Santa Maria, PR. AR: area reforested in 2003.

por parcela, em covas de 20 x 20 x 40 cm e espaçamento de 2 x 2 m(RODRIGUES; LEITÃO FILHO, 2000), aplicado nas entrelinhas do reflorestamento anterior, em 2003. Com base nas análises químicas do solo, adotou-se uma adubação de 80 g de NPK 8-30-10 por cova e 175 g de calcário de conchas por cova.

Foram selecionadas 12 espécies arbóreas com ocorrência natural na região (LORENZI, 2002a, b; BACKES; IRGANG, 2004; CARVALHO, 2006a, b; CARPANEZZI; CARPANEZZI, 2006; GRIS et al., 2012), reunidas em dois grupos ecológicos:

1) Grupo de espécies pioneiras e secundárias iniciais: Trema micrantha (L.) (Blume) (grandiúva), Aloysia virgata (Ruiz \& Pav.) Juss. (lixeira), Cordia americana (L.) Gottschling \& J. S. Mill. (guajuvira), Enterolobium contortisiliquum (Vell.) Morong. (timbori), Peltophorum dubium (Spreng.) Taub. (canafístula) e Bauhinia forficata Link (pata-de-vaca).

2) Grupo de espécies secundárias tardias e climácicas: Jacaranda micrantha Cham. (caroba), Ceiba speciosa (A.St.-Hil.) Ravenna (paineira), Balfourodendron riedelianum (Engl.) Engl. (pau-marfim), Prunus sellowii Koehne (pessegueiro-bravo), Eugenia pyriformis Cambess (uvaia) e Campomanesia xanthocarpa (Mart.) O. Berg (guabiroba).

O plantio ocorreu em dezembro de 2011, e o acompanhamento das mudas foi realizado mensalmente por um ano. Foram considerados os valores de acréscimo

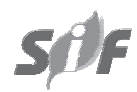

Revista Árvore, Viçosa-MG, v.38, n.6, p.1003-1013, 2014 
mensal em altura e diâmetro à altura do coleto, importante para avaliar a sobrevivência das plantas logo após o plantio (CRUZ et al., 2006). Da mesma forma, a mortalidade das mudas foi registrada a cada mês, a fim de acompanhar o seu estabelecimento.

O manejo sobre o capim-colonião foi realizado mensalmente, bem como as coletas de amostras com a utilização de um gabarito de $50 \times 50 \mathrm{~cm}\left(25 \mathrm{~cm}^{2}\right)$, que era lançado aleatoriamente quatro vezes em cada parcela, a fim de obter uma amostragem final de $1 \mathrm{~m}^{2}$. O capim coletado era seco em estufa a $70^{\circ} \mathrm{C}$ por $48 \mathrm{~h}$ e, então, pesado, avaliando-se, através da massa de matéria seca obtida, a quantidade de capim reincidente a cada mês.

\subsection{Análises do Solo}

Com o intuito de observar as alterações nas características químicas e físicas da área e verificar possíveis alterações nessas características decorrentes do manejo do capim-colonião e plantio das mudas, foram realizadas análises do solo das unidades experimentais.

As amostras foram coletadas em duas profundidades, de $0-10 \mathrm{~cm}$ e de $10-20 \mathrm{~cm}$, de acordo com metodologia da Embrapa (1997), em três épocas, no decorrer do trabalho, sendo a primeira coleta de solo antes do manejo da área (setembro/2011), a segunda no período de implantação das mudas (dezembro/2011) e a terceira 12 meses após a primeira coleta (setembro/ 2012). As características químicas analisadas foram $\mathrm{C}, \mathrm{MO}, \mathrm{H}+\mathrm{Al}$, condutividade elétrica, $\mathrm{P}, \mathrm{K}, \mathrm{Fe}, \mathrm{Ca}, \mathrm{Mg}$, $\mathrm{Na}, \mathrm{Cu}, \mathrm{Mn}$, Zn e capacidade de troca catiônica (CTC). $\mathrm{E}$ as características físicas foram densidade e porosidade do solo (macro e microporosidade).

\subsection{Análises Estatísticas}

Todos os dados coletados foram avaliados quando à homogeneidade, por meio do teste de Cochran. Os dados de desenvolvimento das mudas (crescimento mensal em altura e diâmetro à altura do coleto) e as análises do solo (características químicas e físicas) foram avaliados por meio do teste estatístico ANOVA, em um modelo de parcelas subdivididas. As variáveis massa de matéria seca do capim-colonião e mortalidade das mudas foram submetidas ao teste de ANOVA no modelo unifatorial. Em situações de significância estatística $(p<0,05)$, as variáveis supracitadas foram posteriormente analisadas por meio do teste de Tukey a 5\% de probabilidade. O poder da análise para avaliação do desenvolvimento das mudas foi verificado, tendo sido alcançado um poder de análise de $95 \%$, com tamanho de efeito igual a 0,15 e valores de $\alpha$ e $\beta$ iguais a 0,05 e 0,20 , respectivamente.

\section{RESULTADOS}

A mortalidade verificada nas mudas alcançou um total de 39 indivíduos (24,38\%) e foi estatisticamente significativa ao longo dos meses $(\mathrm{p}<0,05)$ (Tabela 1$)$. Os dois primeiros meses de janeiro $(11,88 \%)$ e fevereiro (5,63\%) apresentaram médias significativamente mais altas. Os demais meses foram semelhantes entre si, com percentuais variando de $0 \%$ a 1,88\%.

Entre as espécies avaliadas, foi observada maior mortalidade das mudas de pessegueiro-bravo logo nos primeiros meses após o plantio. As espécies paineira e a uvaia, no entanto, apresentaram sobrevivência de $100 \%$ dos indivíduos.

Em relação à massa de matéria seca do capimcolonião, houve diferença estatística entre os meses $(p<0,05)$, sendo os maiores valores observados em fevereiro (3,43 g) e dezembro (1,91 g). Contudo, mesmo os maiores valores indicaram que não houve reincidência considerável do capim-colonião ao longo dos meses, o qual variou de 0,04 g a 3,43 g (Tabela 1).

Tabela 1 - Médias de mortalidade das mudas plantadas e massa de matéria seca do capim-colonião no Corredor de Biodiversidade Santa Maria, PR.

Table 1 - Means of planted seedling mortality and dry mass of green panic grass at the Biodiversity Corridor Santa Maria, $P R$.

\begin{tabular}{lcc}
\hline \multicolumn{3}{c}{ MÉDIAS DE TRATAMENTO } \\
\hline & Mortalidade (\%) & Massa seca $\left(\mathbf{g} / \mathbf{m}^{\mathbf{2}}\right)$ \\
\hline $\mathbf{j a n} / \mathbf{1 2}$ & $11,88 \mathrm{a}$ & $1,51 \mathrm{bc}$ \\
$\mathbf{f e v} / \mathbf{1 2}$ & $5,63 \mathrm{ab}$ & $3,43 \mathrm{a}$ \\
$\mathbf{m a r} / \mathbf{1 2}$ & $0,00 \mathrm{~b}$ & $0,07 \mathrm{c}$ \\
abr/12 & $0,63 \mathrm{~b}$ & $0,16 \mathrm{c}$ \\
$\mathbf{m a i} / \mathbf{1 2}$ & $1,88 \mathrm{~b}$ & $0,11 \mathrm{c}$ \\
$\mathbf{j u n} / \mathbf{1 2}$ & $1,88 \mathrm{~b}$ & $0,07 \mathrm{c}$ \\
jul/12 & $0,63 \mathrm{~b}$ & $0,04 \mathrm{c}$ \\
ago/12 & $0,00 \mathrm{~b}$ & $0,23 \mathrm{c}$ \\
$\mathbf{s e t} / \mathbf{1 2}$ & $0,00 \mathrm{~b}$ & $0,29 \mathrm{c}$ \\
out/12 & $0,63 \mathrm{~b}$ & $0,61 \mathrm{bc}$ \\
nov/12 & $0,00 \mathrm{~b}$ & $1,26 \mathrm{bc}$ \\
dez/12 & $1,25 \mathrm{~b}$ & $1,91 \mathrm{ab}$ \\
\hline
\end{tabular}

Médias seguidas da mesma letra não diferem estatisticamente entre si, pelo teste de Tukey a $5 \%$ de significância $(p<0,05)$. Means followed by the same letter do not differ significantly by the Tukey test at a significance level of $5 \%(p<0.05)$. 
Em relação à avaliação do crescimento das mudas em diâmetro e altura, foi possível observar que houve diferenças estatísticas significativas entre as espécies $(\mathrm{p}<0,05)$ (Figura 2). Entre as espécies pertencentes ao grupo 1, a lixeira apresentou os maiores valores de acréscimo em diâmetro $(0,80 \mathrm{~mm})$ e em altura $(5,80 \mathrm{~cm})$. Outras espécies de crescimento intermediário, com bom desenvolvimento em altura, foram timbori e grandiúva, com 3,67 cm e 2,69 cm, respectivamente (Figura 2).

Entre as espécies do grupo 2, a paineira apresentou o maior acréscimo em diâmetro sobre as demais espécies (0,60 mm). Em relação à altura, os maiores valores foram

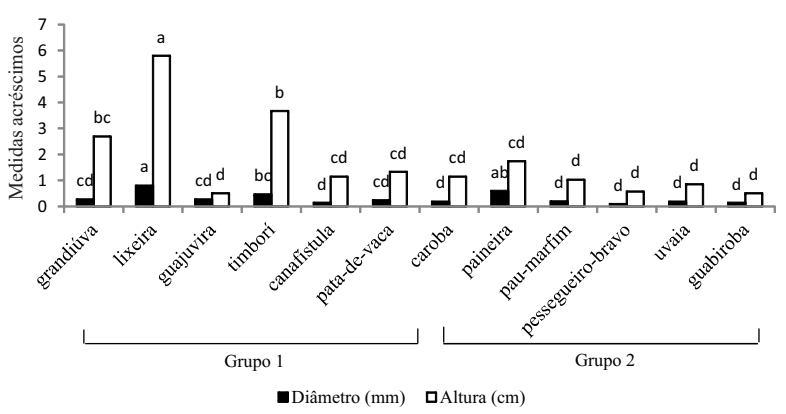

Figura 2 - Médias de crescimento em diâmetro (mm) e altura $(\mathrm{cm})$ das espécies plantadas no Corredor de Biodiversidade Santa Maria, PR. Médias seguidas da mesma letra não diferem estatisticamente entre si, pelo teste de Tukey a 5\% de significância $(\mathrm{p}<0,05)$.

Figure 2 - Means of diameter ( $\mathrm{mm}$ ) and height (cm) growth of the species planted at the Biodiversity Corridor Santa Maria, PR. Means followed by the same letter do not differ significantly by the Tukey test at a significance level of $5 \%(p<0.05)$. verificados na paineira $(1,74 \mathrm{~cm})$ novamente e na caroba $(1,15 \mathrm{~cm})$. Contudo, essas plantas não diferiram estatisticamente das demais (Figura 2).

Da mesma forma, verificou-se sobre o crescimento das mudas interação estatística significativa entre as espécies e os meses $(p<0,05)$. Em relação à variável diâmetro, apenas lixeira, guajuvira e paineira apresentaram variação significativa de crescimento ao longo dos meses; quanto à altura, grandiúva, lixeira, timbori, caroba e paineira apresentaram variação significativa.

Com exceção da lixeira e paineira, foi possível observar que todas apresentaram padrão de crescimento similar ao longo dos meses. Essas duas espécies, por sua vez, pertencentes aos grupos 1 e 2, respectivamente, exibiram um padrão de crescimento similar em relação ao diâmetro, mas, quanto à altura, verificou-se maior amplitude de variação entre as duas espécies (Figura 3).

Ao avaliar as características químicas e físicas do solo sobre a interação entre as profundidades de 0-10 cm e 10-20 cm e as três coletas realizadas (setembro/ 2011, dezembro/2011 e setembro/2012), apenas poucas variáveis apresentaram médias estatisticamente diferentes $(\mathrm{p}<0,05)$. Contudo, quando as características do solo são avaliadas em relação às duas profundidades, apenas a $\mathrm{H}+\mathrm{Al}, \mathrm{Na}, \mathrm{K}$, Fe e a macroporosidade não apresentaram variação significativa $(\mathrm{p}<0,05)$ (Tabela 2$)$. Da mesma forma, as coletas realizadas em três épocas revelaram alterações ocorridas no solo sobre todas as características químicas e físicas ( $\mathrm{p}<0,05)$, com exceção do $\mathrm{Na}$ (Tabela 2). Esses resultados sugerem que as alterações promovidas na área, como a retirada do capim-colonião e o plantio das mudas, podem ter sido capazes de modificar as características do solo ao longo do experimento.

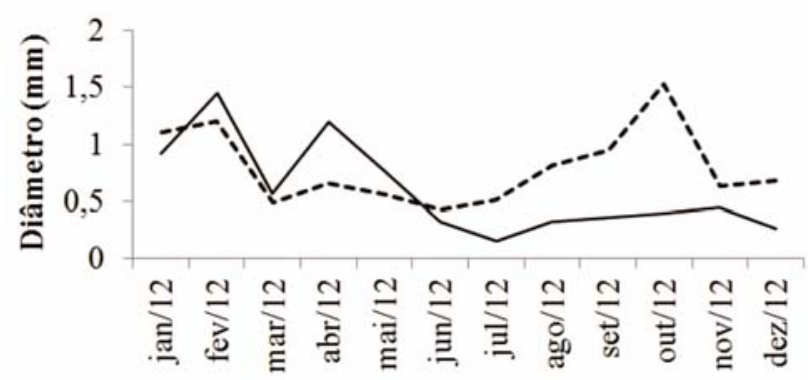

A --- lixeira - paineira

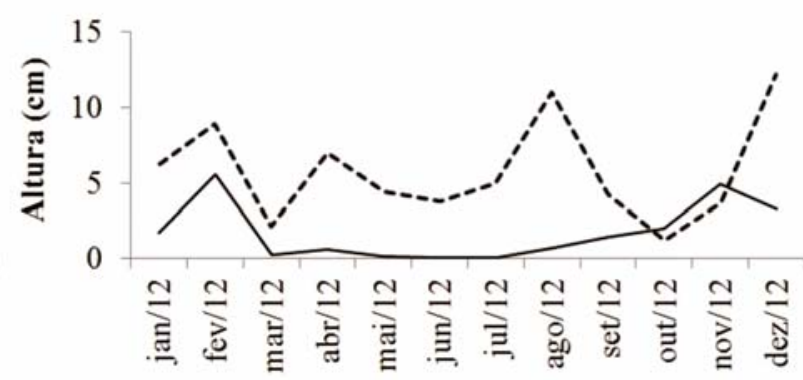

B --- lixeira - paineira

Figura 3 - Médias mensais de crescimento em diâmetro (mm) (A) e altura (cm) (B) das espécies dos grupo 1 e 2 com maior crescimento no Corredor de Biodiversidade Santa Maria, PR.

Figure 3 - Monthly means of diameter ( $\mathrm{mm}$ ) (A) and height growth (cm) (B) of species from groups 1 and 2 with greater growth at the Biodiversity Corridor Santa Maria, PR. 
Tabela 2 - Resultados das análises químicas e físicas do solo no Corredor de Biodiversidade Santa Maria, PR, das coletas realizadas em setembro de 2011 (coleta 1), dezembro de 2011 (coleta 2) e setembro de 2012 (coleta 3), nas profundidades de 0 a $10 \mathrm{~cm}$ e 10 a $20 \mathrm{~cm}$.

Table 2 - Results of chemical and physical analyzes of soil at the Biodiversity Corridor Santa Maria, PR, from samples collected in September 2011 (collect 1), December 2011 (collect 2) and September 2012 (collect 3), at depths of 0 to $10 \mathrm{~cm}$ and 10 to $20 \mathrm{~cm}$.

\begin{tabular}{|c|c|c|c|c|c|c|}
\hline & \multirow[t]{2}{*}{ CARACTERÍSTICAS } & \multicolumn{2}{|c|}{ PROFUNDIDADES } & \multicolumn{3}{|c|}{ COLETAS } \\
\hline & & 0-10 & $10-20$ & Coleta 1 & Coleta 2 & Coleta 3 \\
\hline \multirow[t]{15}{*}{ QUÍMICAS } & $\mathbf{p H}$ & $5,380 a$ & $5,138 b$ & $5,243 b$ & $5,520 a$ & $5,016 \mathrm{c}$ \\
\hline & $\mathrm{H}+\mathrm{Al}\left(\mathrm{Cmolc} / \mathrm{dm}^{3}\right)$ & $4,301 \mathrm{a}$ & $4,290 a$ & $4,658 \mathrm{a}$ & $4,591 \mathrm{a}$ & $3,648 b$ \\
\hline & Na (Cmolc/dm³) & $0,197 \mathrm{a}$ & $0,172 \mathrm{a}$ & $0,188 \mathrm{a}$ & $0,191 \mathrm{a}$ & $0,175 a$ \\
\hline & CE (dS/m) & $2,248 \mathrm{a}$ & $2,189 b$ & 2,291b & $2,370 a$ & $1,996 c$ \\
\hline & $C\left(g / d^{3}\right)$ & $23,354 a$ & $20,453 b$ & $21,878 \mathrm{ab}$ & $22,843 a$ & $20,990 b$ \\
\hline & MO $\left(\mathrm{g} / \mathbf{d m}^{3}\right)$ & $40,261 \mathrm{a}$ & $35,260 b$ & $37,720 a b$ & 39,376a & $36,186 b$ \\
\hline & $P\left(\mathbf{m g} / \mathbf{d m}^{3}\right)$ & $0,223 a$ & $0,170 b$ & $0,173 b$ & $0,284 a$ & $0,132 b$ \\
\hline & $\mathrm{K}\left(\mathrm{Cmolc} / \mathrm{dm}^{3}\right)$ & $1,193 \mathrm{a}$ & $1,286 a$ & $1,667 b$ & $0,994 b$ & $1,055 b$ \\
\hline & Ca (Cmolc/dm³) & $11,239 a$ & $10,125 b$ & $9,261 b$ & $10,221 b$ & $12,564 a$ \\
\hline & $\operatorname{Mg}\left(\mathrm{Cmolc} / \mathrm{dm}^{3}\right)$ & $2,563 a$ & 2,233b & $2,528 \mathrm{a}$ & $2,203 b$ & $2,464 \mathrm{a}$ \\
\hline & Fe $\left(\mathrm{Cmolc} / \mathrm{dm}^{3}\right)$ & $0,531 \mathrm{a}$ & $0,494 a$ & 0,899a & $0,348 b$ & $0,290 b$ \\
\hline & Cu (Cmolc/dm $\left.{ }^{3}\right)$ & $0,025 b$ & $0,028 \mathrm{a}$ & $0,017 \mathrm{c}$ & $0,035 a$ & $0,028 b$ \\
\hline & $\operatorname{Mn}\left(\mathrm{Cmolc} / \mathrm{dm}^{3}\right)$ & $0,302 \mathrm{a}$ & $0,294 b$ & $0,284 b$ & $0,287 b$ & $0,323 a$ \\
\hline & Zn (Cmolc/dm $\left.{ }^{3}\right)$ & $0,027 \mathrm{a}$ & $0,022 b$ & $0,034 a$ & $0,021 b$ & $0,017 \mathrm{c}$ \\
\hline & СТC & $19,297 a$ & $17,934 b$ & $18,106 b$ & $18,008 \mathrm{~b}$ & $19,732 \mathrm{a}$ \\
\hline \multirow[t]{4}{*}{ FÍSICAS } & DS $\left(\mathrm{g} / \mathrm{cm}^{3}\right)$ & $1,158 b$ & $1,267 \mathrm{a}$ & $1,185 b$ & $1,191 \mathrm{~b}$ & $1,261 \mathrm{a}$ \\
\hline & MIC (\%) & $39,202 \mathrm{a}$ & 33,950b & 39,934ab & $38,555 a$ & $34,236 b$ \\
\hline & MAC (\%) & $30,805 a$ & $33,145 a$ & $35,511 \mathrm{a}$ & $34,135 a$ & $26,279 b$ \\
\hline & PT (\%) & $70,006 a$ & $67,097 \mathrm{~b}$ & $72,449 a$ & $72,690 a$ & $60,515 b$ \\
\hline
\end{tabular}

Médias seguidas da mesma letra não diferem estatisticamente entre si, pelo teste de Tukey a $5 \%$ de significância (p<0,05). Means followed by the same letter do not differ significantly by the Tukey test at a significance level of $5 \%$ ( $p<0.05$ ).

\section{DISCUSSÃO}

De acordo com os resultados observados para mortalidade das mudas, os dois primeiros meses após o plantio apresentaram a maior frequência de perdas (11,67\% e 5,63\%). Como o plantio das mudas ocorreu no final do período chuvoso, essa maior mortalidade pode ter sido ocasionada devido à ocorrência de vários dias seguidos de estiagem, observados tanto em dezembro quanto em janeiro.

Em situação de déficit hídrico, os primeiros meses são os mais críticos para o estabelecimento das plantas, levando ao ponto de murcha em espécies mais sensíveis, o que geralmente acontece dentro de quatro a seis dias após o plantio (RODIGUES et al., 2009). Com isso, a área de captação de luz, as reações fotossintéticas e as trocas gasosas são severamente influenciadas (LARCHER, 2006), ocasionando maior mortalidade das mudas.

O pessegueiro-bravo, no qual foi observada alta mortalidade nos primeiros meses, apresentou abscisão foliar em todos os indivíduos, caracterizando situação de estresse hídrico. Por se tratar de espécie mais tardia, na sucessão tende a ser mais sensível à falta de água, com efeitos mais prolongados sobre a espécie (RIBEIRO et al., 2001). Entretanto, a alta sobrevivência observada na paineira pode ter ocorrido em função do seu bom desenvolvimento em diâmetro, uma vez que essa característica está relacionada ao acúmulo de reservas, assegurando maior resistência e fixação ao solo, o que por sua vez garante seu melhor estabelecimento (STURION; ANTUNES, 2000). Além disso, é espécie pouco exigente quanto às características físicas do solo (CARVALHO, 2006a), tendo apresentando alta sobrevivência também em outros trabalhos (MOREIRA, 2004; NICODEMO et al., 2009; GARRIDO et al., 2013).

Da mesma forma, a uvaia, espécie climácica de crescimento lento (LORENZI, 2002a), apresentou sobrevivência de todos os indivíduos, o que sugere tratar de espécie resistente a períodos de estiagem, com boa adaptação às condições ambientais locais, apresentando crescimento similar ao longo dos meses.

Em relação ao controle efetuado sobre o capimcolonião, observou-se que este não apresentou

Revista Árvore, Viçosa-MG, v.38, n.6, p.1003-1013, 2014 
reincidência significativa ao longo dos meses. O manejo da área, associado ao sombreamento promovido pelo reflorestamento realizado em 2003, possivelmente levou a um desgaste em seu banco de sementes, reduzindo sua regeneração.

A permanência do capim dificulta a manutenção do banco de plântulas da área e o estabelecimento de propágulos alóctones, o que pode vir a comprometer a perpetuação da área no longo prazo (QUESTAD; FOSTER, 2008). Além disso, a presença do capim-colonião por tempo prolongado pode favorecer a compactação do solo (HOLL, 1999), com efeito superior ao do pisoteio bovino (LANZANOVA, 2007).

Em relação ao crescimento das mudas, verificou-se que a lixeira apresentou crescimento superior entre as espécies do grupo 1. A lixeira é pioneira, de grande plasticidade fenotípica (CORRÊA, 2003) e, por isso, com ocorrência, inclusive, em áreas com deficiências nutricionais do solo e ambientes sombreados, o que possivelmente favoreceu o seu melhor desenvolvimento na área em relação às demais espécies desse grupo.

Outras espécies do grupo 1 que apresentaram crescimento intermediário em altura foram timbori e grandiúva. O timbori é espécie pioneira que possui boa adaptação a diferentes tipos de solo e até mesmo a solos com baixa fertilidade química, podendo, por isso, ter sido uma das espécies com bom desenvolvimento neste estudo, em que a área apresenta algumas deficiências como pH ácido, o que resulta na limitação da disponibilidade de nutrientes essenciais. Além disso, tolera solos secos, sendo comum em clareiras e capoeirões, com regeneração acentuada (CARVALHO, 2006a). Da mesma forma, a grandiúva apresenta grande versatilidade ambiental, com crescimento similar durante todo o período avaliado, além de ser bastante apreciada pela avifauna, sendo por isso de grande valor ecológico (AMORIM et al., 2006).

As demais espécies dos grupos 1 e 2 apresentaram, em grande parte dos meses analisados, ausência de crescimento ou até diminuição por perda de umidade e das folhas, o que pode indicar situações de estresse (NASCIMENTO et al., 2011; LEMOS et al., 2011).

Tal fato pode estar relacionado aos fatores bióticos e abióticos, como características intrínsecas de cada espécie que possuem diferentes estratégias para estabelecimento em cada tipo de ambiente. Por se tratar de área onde já existiam outras espécies arbóreas, a ação de efeitos alelopáticos e a competição por nutrientes, luz e espaço podem ter contribuído para o menor crescimento das mudas plantadas. Além disso, plantas submetidas à restrição hídrica apresentam redução de crescimento devido à menor turgescência das células, o que influencia no alongamento, diferenciação celular e desenvolvimento da área foliar. Com isso, a produção e translocação de fotoassimilados são afetadas, o que, além da ausência de crescimento, pode levar a reduções na altura, número de folhas e diâmetro do caule (LARCHER, 2006).

Da mesma forma, as características do solo possuem papel importante no desenvolvimento das plantas (WARDLE et al., 2004). Em relação às características físicas, foi observado aumento da densidade do solo ao longo das três coletas devido à redução da razão entre macroporos e microporos (REICHERT et al., 2007).

Em solos compactados, observa-se aumento do conteúdo volumétrico de água e da capacidade de campo, enquanto a aeração e a taxa de infiltração de água e a condutividade hidráulica são reduzidas. Dessa forma, o desenvolvimento do sistema radicular fica comprometido, e há menor aporte de oxigênio disponível na rizosfera e absorção dos nutrientes disponíveis (QUEIROZ-VOLTAN et al., 2000).

Solos com baixo pH também interferem na disponibilidade de nutrientes importantes. A área antes do manejo já apresentava pH ácido (LANTMANN et al., 1982), com redução após o corte do capim-colonião. A rápida decomposição do capim-colonião pode ter promovido a liberação de nutrientes e ácidos orgânicos, levando à acidificação do meio. Além disso, a liberação de exsudatos ácidos pelas raízes das plantas já existentes na área contribui com a redução dos valores de $\mathrm{pH}$ do solo, semelhante ao que foi observado nos trabalhos de Barreto et al. (2006) e Longo e Espíndola (2000).

As concentrações de P no solo são influenciadas pelo pH, e, à medida que este diminui abaixo de 6,0, a disponibilidade de P é reduzida (LANTMANN et al., 1982). Isso inviabiliza o desenvolvimento vegetal, podendo levar a planta à morte (LOPEZ, 1987). Na área de estudo foram observadas concentrações muito baixas desse nutriente, com valores ainda mais reduzidos na terceira coleta de solo, corroborando a relação direta entre pH e P.

Da mesma forma, o Ca e Mg também são afetados indiretamente, uma vez que o $\mathrm{pH}$ age sobre os níveis de $\mathrm{Al}$ e este sobre a disponibilidade desses nutrientes,

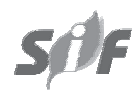

Revista Árvore, Viçosa-MG, v.38, n.6, p.1003-1013, 2014 
os quais, apesar disso, apresentaram-se com concentrações mais elevadas nas duas últimas análises, o que pode estar relacionado à CTC do solo (LANTMANN et al., 1982).

As concentrações de $\mathrm{C}$ e MO sofreram pouca alteração ao longo das coletas, pois apesar da remoção do capim-colonião, que contribuía com a entrada constante de MO no solo, a área apresenta acúmulo de serapilheira que parece estar suprindo esse papel. Apesar disso, a condutividade elétrica encontrada, que indica a quantidade de sais presentes em solução no solo, foi alta nos solos da região e variou de 2,00 $\mathrm{dS} / \mathrm{m}$ a $2,37 \mathrm{dS} / \mathrm{m}$, sendo o menor valor observado na última análise. Solos salinos exigem maior dispêndio de energia da planta para a absorção de água, afetando, dessa forma, processos metabólicos essenciais e prejudicando a germinação e desenvolvimento das mudas (BRANDÃO; LIMA, 2002).

Em relação às profundidades estudadas, não foi verificado efeito significativo sobre as três coletas de solo na maioria das características avaliadas, demonstrando que os resultados das três análises foram independentes das duas profundidades. De forma geral, os nutrientes apresentaram valores reduzidos na camada de 10-20 cm em relação à camada de $0-10 \mathrm{~cm}$, sendo isso esperado, uma vez que a atividade de ciclagem de nutrientes é menor nas camadas mais profundas do solo (NETO et al., 2001).

\section{CONCLUSÃO}

As espécies Aloysia virgata (Ruiz \& Pav.) Juss. (lixeira) e Ceiba speciosa (A. St. -Hil.) Ravenna (paineira) apresentaram maior incremento dentro dos grupos 1 e 2, respectivamente, sendo indicadas para programas de recuperação de áreas degradas nessa tipologia florestal.

O controle do capim-colonião pela metodologia aplicada foi eficiente e de fundamental importância na indução da regeneração natural.

O desenvolvimento moderado das mudas pode ser reflexo de solo compactado, indicando a necessidade de revolvimento do solo para novos plantios.

A recuperação da área, mesmo que por período curto, mostrou-se eficiente na alteração das características físicas e químicas do solo analisadas, bem como a correção do solo, baseada em análises prévias, é essencial na geração de condições adequadas ao desenvolvimento das mudas.
A predominância de espécies iniciais da sucessão ecológica em uma área reflorestada aponta para a necessidade de enriquecimento com espécies tardias, que poderiam auxiliar também no manejo do capimcolonião, viabilizando, por sua vez, a perpetuação do corredor no longo prazo.

\section{AGRADECIMENTOS}

À Administração da Fazenda Santa Maria, por permitir a realização deste Projeto; à equipe do Laboratório de Botânica e PGEAGRI da Unioeste e aos alunos do Curso de Biologia da Uniamérica, pelo auxílio em campo e nas análises; ao Instituto Maytenus, pelo auxílio financeiro; e à ITAIPU Binacional, pela doação das mudas nativas; e à Coordenação de Aperfeiçoamento de Pessoal de Nível Superior (CAPES), pela concessão da bolsa de mestrado à primeira autora .

\section{REFERÊNCIAS}

AMORIM, I. L.; FERREIRA, R. A.; DAVIDE, A. C.; CHAVES, M. M. F. Aspectos morfológicos de plântulas e mudas de Trema. Revista Brasileira de Sementes, v.28, n.1, p.86-91, 2006.

BACKES, P.; IRGANG, B. Árvores do Sul: guia de identificação \& interesse ecológico. Rio de Janeiro: Instituto Souza Cruz, 2004. 204p.

BARRETO, A. C.; LIMA, F. H. S.; FREIRE, M. B. G. S.; ARAÚJO, Q. R.; FREIRE, R. J.

Características químicas e físicas de um solo sob floresta, sistema agroflorestal e pastagem no sul da Bahia. Revista Caatinga, v.19, n.4, p.415-125, 2006.

BRANDÃO, S. L.; LIMA, S. C. pH e condutividade elétrica em solução do solo, em áreas de pinus e cerrado na Chapada, em Uberlândia (MG) . Caminhos de Geografia, v.3, n.6, p.46-56, 2002.

CABIN, R. J.; WELLER, S. G.; LORENCE, D. H.; CORDELL, S.; HADWAY, L. J. Effects of microsite, water, weeding, and direct seeding on the regeneration of native and alien species within a Hawaiian dry forest preserve.

Biological Conservation, v.104, n.2, p.181190, 2002. 
CARPANEZZI, A. A.; CARPANEZZI, O. T. B. Espécies nativas recomendadas para a recuperação ambiental no Estado do Paraná, em solos não degradados. Colombo: Embrapa Florestas, 2006.

CARVALHO, P. E. R. Espécies arbóreas brasileiras. Colombo: Embrapa Florestas, 2006a. v.1. 1039p.

CARVALHO, P. E. R. Espécies arbóreas brasileiras. Colombo: Embrapa Florestas, 2006b. v.1. 627p.

CORRÊA, I. P. Plasticidade fenotípica em indivíduos jovens de Aloysia virgata (Ruiz \& Pav.) Juss. - Verbenaceae. 2003. 58f. Dissertação (Mestrado em Ecologia e Recursos Naturais) - Universidade Federal de São Carlos, São Carlos, 2003.

CRUZ, C. A. F.; PAIVA, H. N.; GUERRERO, C. R. A. Efeito da adubação nitrogenada na produção de mudas de sete-cascas (Samanea inopinata (Harms) Ducke). Revista Árvore, v.30, n.4, p.537-546, 2006.

DAEHLER, C. C. Performance comparisons of cooccurring native and alien invasive plants: implications for conservation and restoration.

Annual Review of Ecology, Evolution, and Systematics, v.34, p.183-211, 2003.

EMPRESA BRASILEIRA DE PESQUISA

AGROPECUÁRIA - EMBRAPA. Centro Nacional de Pesquisa de Solos. Manual de métodos e análise de solo. 2.ed. Rio de Janeiro: 1997. 212p.

FREIRE, O. Solos das regiões tropicais. Botucatu: FEPAF, 2006.

GALINDO-LEAL, C.; CÂMARA, G. I. Status do hotspot Mata Atlântica: uma síntese. In: GALINDO-LEAL, C.; CÂMARA, G. I. (Ed.) The Atlantic Forest of South America: biodiversity status, threats, and outlook. Washington: Island Press, 2003. p.3-11.

GANEM, R. S. Corredores ecológicos - o que são?. Brasília, DF: Consultoria Legislativa da Câmara dos Deputados, 2005. Disponível em: <http://bd.camara.gov.br/bd/bitstream/handle/ bdcamara/1263/ corredores_ecologicos_senna.pdf?sequence=1> Acesso em: 20 mar. 2011.

GARRIDO, M. A. O.; FARIA, H. H.; DURIGAN, G.; CONTIÉRI, W. A.; KAWABATA, M. Recomposição de Mata Ciliar em microbacia hidrográfica, SP, Brasil. Disponível em: <http://www.secforestales.org/ buscador/pdf/2CFE02-057.pdf $>$ Acesso em: 15 mar. 2013.

GRIS, D.; TEMPONI, L. G.; MARCON, T. R. Native species indicated for degraded area recovery in Western Paraná, Brazil. Revista Árvore, v.36, n.1, p.113-125, 2012.

GROENEVELDA, J.; ALVES, L. F.; BERNACCI, L. C.; CATHARINO, E. L. M.; KNOGGE, C.; METZGER, J. P.; PÜTZ, S.; HUTH, A. The impact of fragmentation and density regulation on forest succession in the Atlantic rain forest.

Ecological Modelling, v.220, n.19, p.24502459, 2009.

HOLL, K. D. Factors limiting tropical rain forest regeneration in abandoned pasture: seed rain, seed germination, microclimate, and soil.

Biotropica, v.31, n.2, p.229-242, 1999.

HOLL, K. D. Do bird perching structures elevate seed rain and seedling establishment in abandoned tropical pasture? Restoration Ecology, v.6, n.3, p.253-261, 2002.

INSTITUTO AGRONÔMICO DO PARANÁ IAPAR. Cartas climáticas do Paraná. Disponível em: <http://www.iapar.br/modules/ conteudo/conteudo.php?conteudo $=863>$ Acesso em: 26 jun. 2011.

\section{INSTITUTO BRASILEIRO DE GEOGRAFIA E} ESTATÍSTICA - IBGE. Departamento de Recursos Naturais e Estudos Ambientais. Manual técnico da vegetação brasileira. Rio de Janeiro: 2012. 92p.

LANTMANN, A. F.; OLIVEIRA, E. L.; PALHANO, J. B.; CHAVES, J. C. D.; VOSS, M.; PARRA, M. S.; MUZILLI, O.; CAMPOS, R. J. Bases para o uso racional de corretivos a adubos no Estado do Parana. Londrina: IAPAR/EMBRAPA-CNPSo, 1982. 184p.

Revista Árvore, Viçosa-MG, v.38, n.6, p.1003-1013, 2014 
LANZANOVA, M. E.; NICOLOSO, R. D. S.; LOVATO, T.; ELTZ, F. L. F.; AMADO, T. J. C.; REINERT, D. J. Atributos físicos do solo em sistema de integração lavoura-pecuária sob plantio direto. Revista Brasileira de Ciência do Solo, v.31, p.1131-1140, 2007.

LARCHER, W. Ecofisiologia vegetal. São Carlos: Rima, 2006.

LEMOS, A. O.; SILVA, S. I.; PIMENTEL, R. M. M.; XAVIER, B. T.; ARAÚJO, E. L. Respostas ecofisiológicas de Bernardia sidoides Müll. Arg. ao estresse hídrico. Revista de Geografia, v.28, n.1, p.149-160, 2011.

LONGO, R. M.; ESPÍNDOLA, C. R. Alterações em características químicas de solos da região Amazônica pela introdução de pastagens. Acta Amazonica, v.30, n.1, p.71-80, 2000.

LOPEZ, R. J. El diagnóstico de suelos y plantas (métodos de campo y laboratorio. Madrid: Mundi-Prensa Libros, 1987. 363p.

LORENZI, H. Árvores brasileiras: manual de identicação e cultivo de plantas arbóreas do Brasil. 4.ed. Nova Odessa: Instituto Plantarum, 2002a. v.1. 368p.

LORENZI, H. Árvores brasileiras: manual de identicação e cultivo de plantas arbóreas do Brasil. 4.ed. Nova Odessa: Instituto Plantarum, 2002b. v.2. 367p.

MARTINS, S. V. Recuperação de Matas Ciliares. 2.ed. Viçosa, MG: CPT, 2007. 255p.

MINEROPAR. Minerais do Paraná. Geologia do Paraná. 2008. Disponível em: <http:// www.mineropar.pr.gov.br/modules/conteudo/ conteudo.php?conteudo=106 $>$ Acesso em: 28 jan. 2013.

MONACO, T. A.; SHELEY, R. L. Invasive plant ecology and management: linking processes to practice. Wallingford: Cabi Publishing, 2012. v.2. 216p.

MOREIRA, P. R. Manejo do solo e recomposição da vegetação com vistas a recuperação de áreas degradadas pela extração de bauxita, Poços de Caldas, MG. 2004. 139f. Tese (Doutorado em Ciências Biológicas) - Universidade Estadual Paulista”Julio de Mesquita Filho, Rio Claro, 2004.

NASCIMENTO, H. H. C. et al. Análise do crescimento de mudas de jatobá (Humenaea courbaril L.) em diferentes níveis de água no solo. Revista Árvore, v.35, n.3, p.617-626, 2011. (Edição Especial)

NEGREIROS, D.; FERNANDES, G. W.; BERBARA, R. L. L.; RODARTE, L. H. O.; BARBOSA, N. P. U. Physicochemical characterization of quartzitic degraded soils and adjacent areas of rupestrian fields, Serra do Cipó, MG, Brazil. Neotropical Biology and Conservation, v.6, n.3, p.156161, 2011.

NICODEMO, M. L. F.; PORFIRIO-DA-SILVA, V.; SANTOS, M.; VINHOLIS, M. M. B.; FREITAS, A. R.; CAPUTTI, G. Desenvolvimento inicial de espécies florestais em sistema silvipastoril na região sudeste. Pesquisa Florestal

Brasileira, n.60, p.89-92, 2009.

PÜTZ, S.; GROENEVELD, J.; ALVES, L. F.; METZGER, J. P.; HUTH, A. Fragmentation drives tropical forest fragments to early successional states: A modelling study for Brazilian Atlantic forests. Ecological Modelling, v.222, n.12, p.1986-1997, 2011.

QUEIROZ-VOLTAN, R. B.; NOGUEIRA, S. S. S.; MIRANDA, M. A. C. Aspectos da estrutura da raiz e do desenvolvimento de plantas de soja em solos compactados. Pesquisa

Agropecuária Brasileira, v.35, n.5, p.929938, 2000.

QUESTAD, E. J.; FOSTER, B. L. Coexistence through spatiotemporal heterogeneity and species sorting in grassland plant communities. Ecology Letters, v.11, n.7, p.717-726, 2008.

REICHERT, J. M.; SUZUKI, L. E. A. S.; REINERT, D. J. Compactação do solo em sistemas agropecuários e florestais: identificação, efeitos, limites críticos e mitigação. In: CERETTA, C. A.; SILVA, L. S.; REIHCERT, J. M.,

(Ed.) Tópicos em ciência do solo. Viçosa, MG: Sociedade Brasileira de Ciência do Solo, 2007. v.5. p.49-134. 
RIBEIRO, J. F.; FONSECA, C. E. L.; SOUZASILVA, J. C. Cerrado: caracterização e recuperação de matas de galeria. Planaltina: Embrapa Cerrados, 2001.

RIBEIRO, M. C.; METZGER, J. P.; MARTENSEN, A. C.; PONZONI, F. J.; HIROTA, M. M. Brazilian Atlantic forest: how much is left, and how is the remaining forest distributed? Implications for conservation. Biological Conservation, v.142, n.6, p.1141-1153, 2009.

RODRIGUES, R. R.; BRANCALION, P. H. S.; ISEMHAGEN, I. Pacto pela restauração da Mata Atlântica: referencial dos conceitos e ações de restauração florestal. São Paulo: LERF/ ESALQ/Instituto BioAtlântica, 2009. 256p.

RODRIGUES, R. R.; LEITÃO FILHO, H. F. Matas Ciliares: conservação e recuperação. São Paulo: EDUSP, 2000. 320p.

SILVA, J. M. C.; CASTELETI, C. H. M. Status of the biodiversity of the Atlantic Forest of Brazil. In: GALINDO-LEAL, C.; CÂMARA, I. G. (Ed.) The Atlantic Forest of South America: biodiversity status, threats, and outlook.
Washington: Island Press, 2003. p.43-59.

STURION, J. A.; ANTUNES, B. M. A. Produção de mudas de espécies florestais. In: GALVÃO, A. P. M. Reflorestamento de propriedades rurais para fins produtivos e ambientais. Brasília: Embrapa, 2000. p.125-150.

TOSSULINO, M. G. P.; SCHAITZA, E. G.; SIQUEIRA, J. D. P.; SAYAMA, C.; MORATO, S. A. A.; ULANDOWSKI, L. K. M. A.; CAVILHA, M. R. Resumo executivo da avaliação ecológica rápida do corredor IguaçuParaná. Curitiba: Instituto Ambiental do Paraná, 2007. 46p.

VOS, C. C.; BAVECO, H.; GRASHOF-BOKDAM, C. J. Corridors and species dispersal. In:

GUTZWILLER, K. (Ed.) Applying landscape ecology in biological conservation. New York: Springer, 2002. p.84-104.

WARDLE, D. A.; BARDGETT, R. D.; KLIRONOMOS, J. N.; SETÄLÄ, H.; VAN DER PUTTEN, W. H.; WALL, D. H. Ecological linkages between aboveground and belowground biota. Science, v.304, n.5677, p.1629-1633, 2004. 
